\title{
THE USE OF CAVITATING JETS TO OXIDIZE ORGANIC COMPOUNDS IN WATER
}

\author{
K. M. Kalumuck and G. L. Chahine \\ Dynałow, Inc. J essup, MD 20794 \\ email: info@dynałow-inc.com
}

\begin{abstract}
This paper reports on the application of hydrodynamic cavitation by the use of submerged cavitating liquid jets to trigger widespread cavitation and induce oxidation of organic compounds in the bulk liquid solution with a two order of magnitude increase in energy ec ciency compared to the ultrasonic means.

The results are compared to a bubble dynamics model that includes heat and mass transport, collective bubble exects, and a ..rst order Arrhenius reaction rate model. Comparison of model results with experiment indicated the reactions were limited by contaminant transport to the bubble surface rather than by radical generation or the intensity of bubble collapse. Other ..ndings are the desirability of operating at atmospheric ambient pressure and low driving pressures and of maximizing cavity surface area. These results suggest a great potential for the use of jet cavitation in practical scale waste treatment and remediation systems.
\end{abstract}

\section{Introduction}

Overview

Ultrasonic cavitation is known (Brown and Goodman, 1965) to produce sonochemically activated reactions in water resulting in the formation of highly exective oxidizing hydroxyl radicals. Usually this is achieved using ultrasonic horns that send a high intensity acoustic beam into the solution and excite microcavities. Such systems have been found to promote a wide range of chemical reactions (Suslick, 1988) and to be capable of oxidizing dilute aqueous mixtures of organic compounds. However, such devices essentially self limit the ec ciency of the process by achieving cavitation only in a thin layer near the surface of the soni. .er and are not very ec cient. We employ a medhanism for generating cavitation in a wide body of the liquid by an array of submerged cavitating jets (Chahine \& Kalumuck, 2001a\&b; Kalumudk \& Chahine, 2000). This process can be made very ec cient and also bene. ts from the relatively high ec ciencies of pumps.

When subjected to cavitation, water undergoes dissolution according to the following chemical reaction (e.g., Suslick, 1989; Neppiras, 1980):

$$
\mathrm{H}_{2} \mathrm{O} ! \mathrm{H}^{\phi}+\mathrm{OH}^{\phi} \text { : }
$$

The free hydroxyl radical $\mathrm{OH}^{\dagger}$ is one of the most powerful oxidizing agents. Oxidation of organic compounds results in various intermediate and end products depending on the compound. These include water vapor, carbon dioxide, inorganic ions and short chain inorganic acids (e.g., see Suslick, 1988; Hua et al., 1995a; Skov et al., 1997). Intermediate products usually undergo subsequent oxidation. Modeling of radical production due to cavitation bubble collapse has recently been performed by Gong and Hart (1998).

A number of recent studies have used ultrasound to degrade organic contaminants. A partial list includes Hua et al. (1995a, b), Kotronarou et al. (1991), Cheung et al. (1991), and Hua and Hoxman (1996). Such work has been performed in both batch and continuous fow modes using ultrasonic horns and plates. Recently, a commercial process has employed a venturi type cavitation łow loop in combination with UV irradiation and hydrogen peroxide addition (Skov et al., 1997). 


\section{Cavitation Bubble Dynamics}

It is generally accepted that water dissociates under intense insoni..cation due to the resulting growth and collapse of microscopic bubbles. The maximum pressure may be as high as $1.2 \mathrm{f} 10^{4} \mathrm{~atm}$, and the temperature could be about 10,000 ${ }^{ \pm K}$ (Young, 1989): Thus extremely high values of temperature and pressure are generated in a small region of space where the bubble collapse occurs. Such conditions could explain the enhancement by cavitation of the chemical dissociation of the aqueous medium releasing hydroxyl radicals.

However, cavitation bubbles rarely behave spherically. Typically, due to initial or boundary condition asymmetries and to bubble interactions, the bubble, upon collapse, forms a high speed rentering jet. In cavitating jets, elongated, rotating, and ring shaped bubble cavities form which have also been found to collapse with the formation of reentering jets (Chahine and J ohnson, 1985; Chahine and Genoux, 1983).

\section{Cavitating Water J ets}

Cavitating water jet technology represents one successful attempt to harness and utilize the destructive power of cavitation. Various means and nozzle designs can be used to induce the explosive growth of microscopic cavities or bubbles within a liquid jet. Moving away from the ori..ce region, these bubbles encounter higher pressures and collapse (Chahine and J ohnson, 1985; Chahine et al., 1995).

The dimensionless parameter characterizing cavitation is the cavitation number, $3 / 4=\frac{P_{a m b i} p_{v}}{\phi P}$, where $P_{a m b}$ is the ambient liquid pressure, $p$ is the liquid vapor pressure, and $\phi P$ is the pressure drop across the nozzle. The value at which cavitation is incipient is de.ned as the cavitation inception number, $3 / 4:$ If $3 / 43 / 4$ $<1$, cavitation will occur, and as $3 / 43 / 4$ decreases below unity the amount of cavitation will increase.

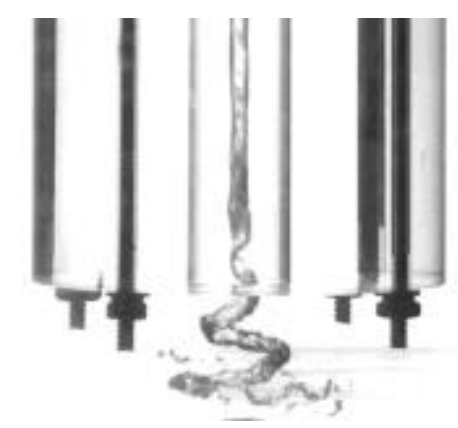

Figure 1: Strobe photograph of plexiglass walled swirling caivtating jet nozzle

The swirling cavitating jet, DynaSwir ${ }^{k}{ }^{k}$, achieves cavitation at very high cavitation numbers (Figure 1). The fow enters a swirl chamber by means of tangential injection slots. The swirl produces a central line vortex which cavitates. As the jet exits the nozzle and impacts on a wall, this ..lament possesses a helicoidal shape with a vortex breakdown at the end of a rotating cork screw shape in the most unstable con. .gurations. For oxidation, this jet has the additional advantage of generating a very large cavity surface area.

\section{Experimental Setup}

Flow Loops and J et N ozzles

Experiments were conducted in several jet łow loops and in an ultrasonic system. Investigations were also conducted in a cavitation reaction chamber constructed of plexiglass to enable viewing of the cavitation and fow. Due to the potential for many organic compounds to attack plexiglass this cell was not used for actual oxidation tests. Instead, jet cavitation reactors constructed of stainless steel were utilized. 


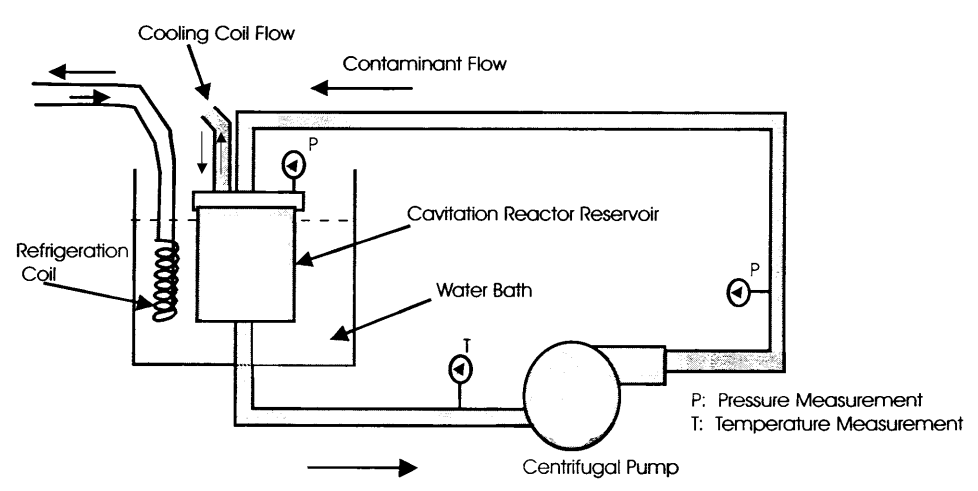

Figure 2: Sketch of łow loop

Figure 2 provides a sketch of the functional con. guration of these test loops. Upon exiting the pump, the liquid fows into a cavitation reaction chamber into which various jet con..gurations can be inserted. The ambient pressure at the jet exit is monitored and controlled as is the pressure of the $\neq$ ow entering the chamber to form the jet(s). Temperature is maintained at the desired value by use of a cooling loop and tank. pH was also monitored and controlled by the addition of small quantities of phosphoric acid or sodium hydroxide. A sampling port/ valve is located in the lid of the reaction chamber or in the piping exiting the chamber.

In addition to the swirling cavitating jet (Figure 1), a multi-ori..ce manifold con. .guration of St $r$ at oJ et cavitating jet nozzles was employed (Kalumuck, et. al. 2000; Chahine \& J ohnson, 1985). The liquid łows into a pipe manifold into which a large number of ori..ces have been machined. The number of ori..ces was varied between 12 and 216. Dixerent manifolds were utilized for dixerent ori..ce diameters between 0.040 and 0.15 in. The manifold was sometimes surrounded by a cylindrical shroud onto which the jet fows were directed to enhance cavitation bubble collapse. The bubbles generated in the jet shear layer collapse in the increased pressure of the jet stagnation region along this sleeve

To observe the cavitation characteristics and dynamics, plexiglass cavitation reaction chambers were used. The loops were operated with pure water at the same conditions as the oxidation experiments with contaminants. The results were recorded utilizing high intensity strobe lighting and a video camera with zoom lens. A series of cavitating vortex rings (Figure $3 b$ ) were distinctly visible in the jet fows in most cases.

Visualization of the swirling cavitating jet (Figure 3a) showed a large volume of cavitation in the form of a line vortex cavity that became vortical upon exiting the ori..ce Typically, a cavity was seen to extend the length of the chamber along its centerline representing the cavitating core of the vortex formed by the swirling fow. As this cavity exited with the jet fow, it developed a rotating helical pattern, broke up, and collapsed.
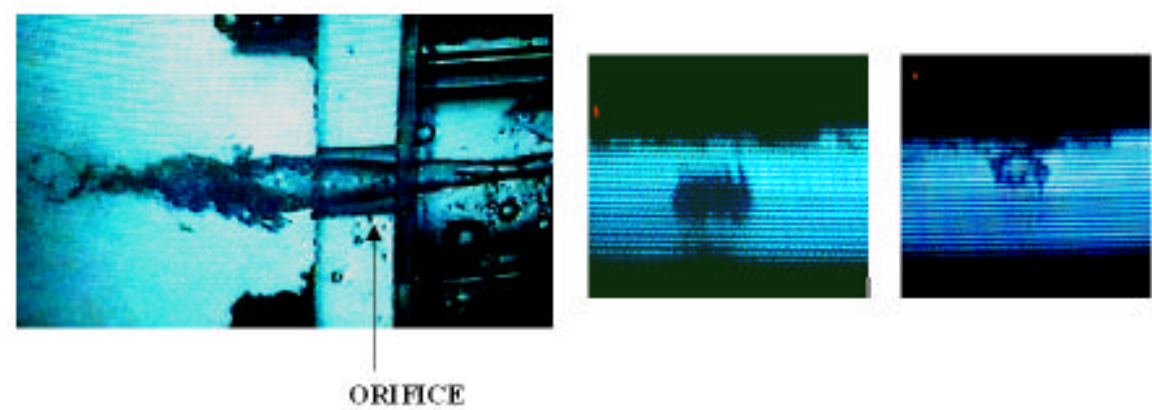

Figure 3: Visualization of cavitation generated by a) swirling jet (left) and b) multiori..ce jet (center \& right showing ring structures) 
M easurement Techniques and Procedures

Two compounds: p-nitrophenol (PNP) and Methyl Orange (MO) were selected. PNP is a phenol related to a number of pesticides and has been studied by others using ultrasonic cavitation enabling comparison with their results. MO is an organic dye that is a nitrogen bearing aromatic compound and contains a sulphonate group, which commonly appears in detergents.

Reagent grade p-Nitrophenol PNP (Aldrich, 99\%), MO, phosphoric acid (Aldrich, 85\%), and sodium hydroxide (VWR Scienti..c, $1.0 \mathrm{~N}$ ) were used. The PNP was in crystalline form and was mixed with distilled water. Contaminant concentrations were measured using a UV-Vis spectrophotometer following the procedures of Kotronarou et al. (1991) and Hua et al. (1995b). The spectrophotometer was calibrated against known concentrations of contaminants in distilled water. During testing, $3 \mathrm{ml}$ samples were drawn from the test reservoir for concentration measurements. Detailed chemical measurement procedures can be found in Kalumuck, et. al. (2000).

\section{Results and Discussion}

\section{Performance Evaluation: Oxidation $\mathrm{E} \pitchfork$ ciency}
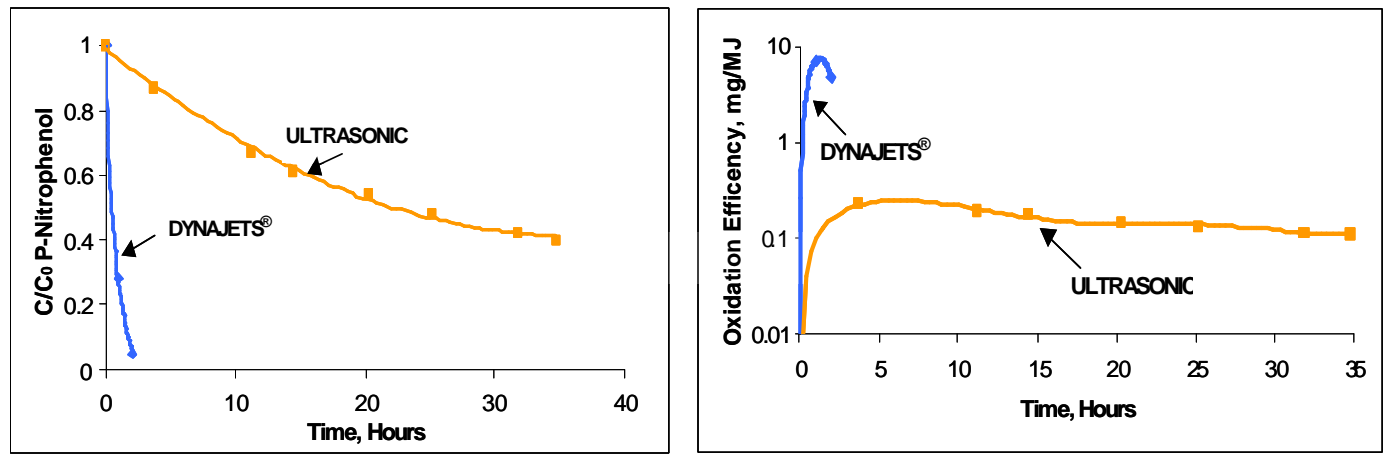

Figure 4: Comparison of concentration reduction and oxidation ec ciencies of cavitating jets and ultrasonic device for PNP

A key measure of the performance of the oxidation process is the energy required to remove a unit mass of a given compound. This can be expressed as the cumulative mass of contaminant removed per unit energy expended. We de. ne this to be the oxidation ec ciency given by:

$$
{ }^{\infty}(\mathrm{t})=\frac{\left(\mathrm{C}_{\mathrm{o}} \mathrm{i} \mathrm{C}(\mathrm{t})\right) \phi \mathrm{V}}{\mathrm{t} \phi \mathrm{P}}:
$$

Here, $C_{0}$ is the initial concentration, $C(t)$ the concentration at timet, $V$ the liquid volume, and $P$ the power expended. The power used in this eq ciency calculation is that which is imparted to the liquid.

Figure 4 compares the concentration reduction histories and oxidation ec ciencies of jet and ultrasonic cavitation. This ..gure presents sample results of the oxidation of PNP (8 ppm initial concentration) with submerged cavitating jets. While the investigations conducted have not as yet been of suф cient scope to state that either the jet or ultrasonic devices are operating at their optima, a range of parameters have been investigated in the current study for the jets and in the literature for the ultrasonic device. The conditions of Figures 4 are near the best known for each device the cavitating jet results exhibit overall energy ec ciencies 100 times higher than the ultrasonic device. This suggests strong promise for application of jet cavitation to oxidation.

\section{Cavitation Number/ A mbient P ressure}

Operation at elevated ambient pressures was found to be less ec cient than operation of the reaction chamber at atmospheric pressure - an unexpected result as cavitation bubbles are known to collapse more strongly at elevated ambient pressures. A potential explanation is provided by the modeling eaort described below 
in which the temperatures of bubble collapse are predicted to be higher at the lower ambient pressures. Figure 5 presents results for ambient pressures between 24 and 100 psig and an initial PNP concentration of approximately $8 \mathrm{ppm}$ with ferric sulfate addition. Both the oxidation rate and ed ciency decline with increasing ambient pressure. Shown are also results for ambient pressures of 50 and 100 psig at an approximately constant value of the cavitation number, sigma $1 / 40.2$. The ec ciency is approximately three times greater at the lower ambient pressure These results have very positive practical implications. There is no need to operate at elevated pressures. Thus the equi pment can be relatively simple with modest capital costs.

PNe Oxidation - Nominal Co: 8ppm; 10 ppem Ferric Sulfste

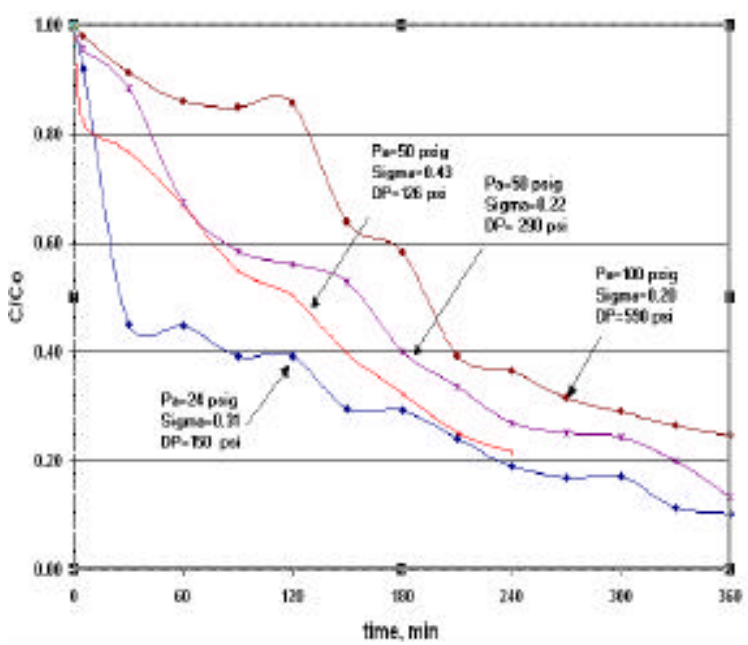

PIIP Oxidation - Nominal Co; 8 ppm: 10 ppm Ferric Sulfato

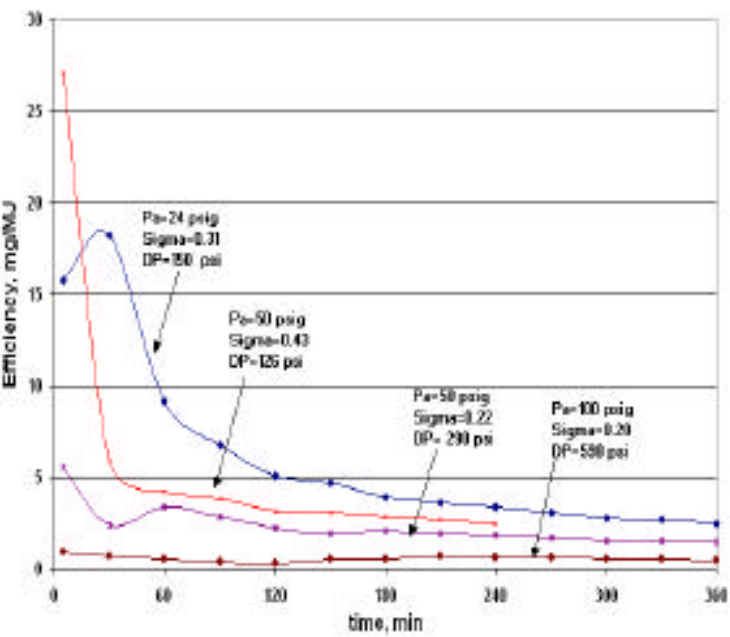

Figure 5: Inłuence of ambient pressure, $\mathrm{Pa}$, and cavitation number, sigma, on oxidation of PNP. a) Concentration histories (left). b) Oxidation ec ciencies (right).

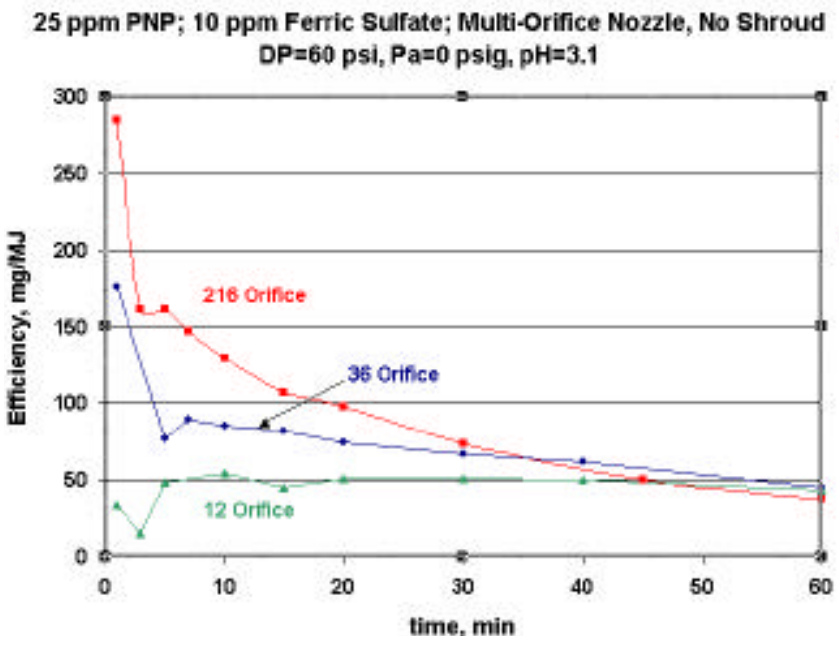

Figure 6: Inłuence of number and size of ori..ces on oxidation eф ciency.

Number and Size of Ori..ces

Figure 6 shows the in $\neq$ uence of the number and size of the ori. .ces for the multi-ori..ce manifold con. .guration for oxidation of PNP. Shown are the results of three dixerent con. .gurations: 12 ori..ces of 0.15 in. diameter, 36 ori. .ces of $0.087 \mathrm{in}$. diameter and 216 ori. .ces of 0.04 in. diameter. All were operated at the same pressures, temperature and $\mathrm{pH}$. The 12 and 36 ori. .ce cases had the same fow rate while the 216 ori. .ce case had an increase in $\ddagger$ ow rate of $25 \%$ due to a corresponding increase in total ori. .ce cross section area. This dixerence is accounted for, however, when the results are normalized by the expended power to calculate the eq ciency 
as done in Figure 6. The results clearly show that more smaller ori..ces, desirable resulting in a increase in the total surface area of the cavities generated, is desirable.

\section{Mechanistic Modeling}

Conceptually, contaminant removal is a multi-step process that includes: 1) radical generation, 2) transport of contaminants and radicals, and 3) reaction between contaminant and radicals. Step 1 is presumably controlled by the bubble dynamics. Step 2 is controlled by the $\neq u$ uid dynamics through viscous and turbulent dixusion and mixing. Step 3 is controlled by the chemical kinetics. The models considered are briefy summarized here More details can be found in Kalumuck, et. al. 2000.

Spherical bubble collapse model

The bubble dynamics for a bubble of radius a is modeled using a modi..ed Rayleigh-Plesset equation that accounts for the liquid pressure change, $₫ \mathrm{P}$; in the jet fow ( $\neq$ owing into a liquid of ambient pressure $p_{a m b}$ ) and the presence of a bubble population obtained from a 1-D bubbly fow model with the liquid density dependent on the local void fraction, $\mathbb{R}$ ?

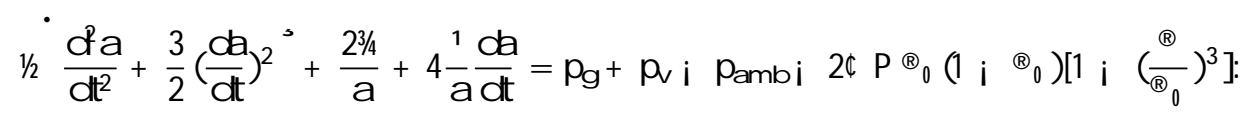

Here, $3 / 4$ is the surface tension, ${ }^{1}$ is the liquid viscosity, ${ }^{1 / 2}$ the liquid density, $p_{g}$ and $p$ the partial pressures of noncondensible gas and vapor within the bubble The subscript 0 refers to equilibrium conditions upstream of the jet ori..ce at a liquid pressure equal to $p_{a m b}+\phi P$ :

The mass and thermal convection/dixusion equations in the liquid are solved using a thin boundary layer approximation (Plesset \& Zwick, 1952) and Henry's Law at the liquid/ cavity interface for each gas component. Vaporization/ condensation occurs at the interface and latent heat is considered in the energy balance (eg., Gumerov, 2000).

The bubble is modeled as containing an ideal gas mixture of vapor and gases. The radicals produced are taken to instantly react with contaminants near the bubble surface Reactions are thus controlled by contaminant transport to the bubble surface and by radical generation, and no signi. .cant concentrations of contaminants or radicals are maintained within the bubble. Radical production occurs in the gaseous phase according to an exponential dependence of the rate on temperature (the commonly used Arrhenius kinetic mechanism; eg., Perry \& Green, 1984) and is proportional to the gas pressure. The reaction is modeled as 1st order with respect to the target compound concentration with a rate constant $\cdot$. We relate $\cdot$ to bubble dynamics through its dependence on the bubble gas temperature and pressure:

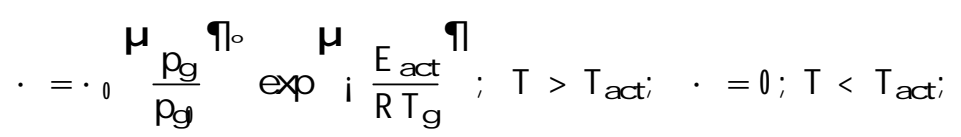

where $E_{\text {act }}$ is the activation energy, $R$ is the universal gas constant, and 0 is a constant related to the stoichiometric coec cient of reaction. This model includes a threshold dependence where $T_{\text {act }}$ is theactivation temperature. The boundary condition at the bubble interface $S$ is:

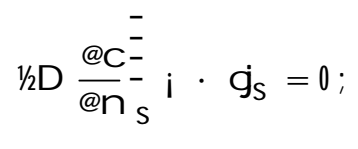

where $1 / 2$ is the liquid density, $c$ is the contaminant concentration, and $D$ is the contaminant dixusivity in the liquid. For an initial uniform contaminant concentration, $c_{0}$, the mass $\neq u x, J$, and the contaminant concentration at the bubble wall, $\mathrm{c}_{\mathrm{a}}$; are:

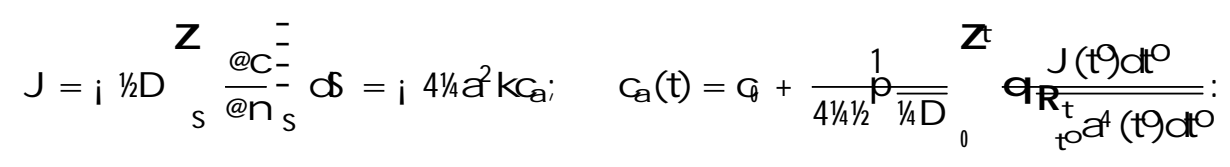


For a multiphase mixture of constant liquid volume $\mathrm{V}$ containing $\mathrm{n}$ bubbles, the void fraction, ${ }^{\circledR}$, and the change in the mass $m$ of component $M$ are:

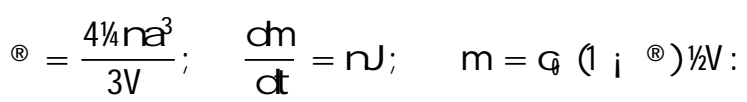

Using h:i to denote the time average of a quantity and noting that $\mathrm{H} \mathrm{i}$ is a function of the initial concentration, i.e, $\mathrm{h} \mathrm{i}=\mathrm{F}\left(\mathrm{c}_{0}\right)$; the decrease of the average concentration is:

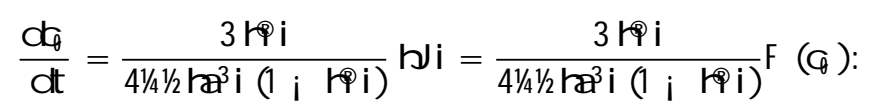

Equation (8) applies when the total system volume equal the volume of the cavitation chamber. For a closed loop with liquid volume $\mathrm{V}$; and a cavitation zone of volume $\mathrm{V}_{\mathrm{Cz}}$; the rate of oxidation for the entire volume is:

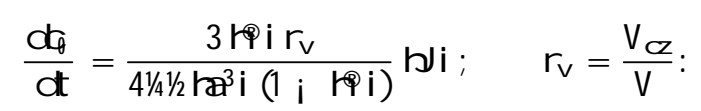

The bulk contaminant concentration decrease due to the presence of many bubbles and recirculation of the liquid in the fow loop is calculated by numerically solving equations (6) and (9) in conjunction with the bubble dynamics (Kalumuck, et. al., 2000).

R esults

A series of runs with varying parameters were made for conditions representative of those for the nozzle con. guration with 216 ori..ces of $0.04 \mathrm{in}$. diameter. The baseline input data was: $\mathrm{k}_{\mathrm{o}}=1000 \mathrm{~kg}=\mathrm{m}^{2}$; liquid dixusivity for PNP in water, $D=2: 4 \pm 10^{9} \mathrm{~m}^{2}=\mathrm{s} ; \mathrm{C}_{0}=10^{5}(10 \mathrm{ppm}) ; T_{\text {act }}=5000{ }^{ \pm} \mathrm{K}$; $~ o=1$; mean void fraction in the cavitation zone, hBi $=0.02$.

Figure 7 illustrates the infuence of the type of noncondensible gas within the bubble: air and argon. Relative to air, argon results in a modestly increased temperature, a shorter period, and a more rapid concentration drop. At $1 \mathrm{hr}$. the air case drops to $20 \%$ of the initial concentration while the Argon case drops to about $8 \%$. Oxidation rate increases due to the presence of an inert gas such as Argon are repeatedly reported in the literature for ultrasonic oxidation. However, our jet experiments showed no signi..cant exects due to the addition of Argon.

Figure 8 shows the in $\neq$ uence of ambient pressure at a constant cavitation number of 0.25 . Since the cavitation number is conserved, the jet velocity is higher in the elevated ambient pressure case resulting in less transit time before impingement on the plate The lower ambient pressure case results in higher temperatures and more rapid oxidation. This is also found experimentally. The power required to operate at twice the pressure (and thus $40 \%$ more fow) is 2.8 times as large. Thus, for $1 \mathrm{hr}$ of operation, the lower pressure case ec ciency is greater by a factor of 3.4 .

\section{In..nite R eaction R ate Limiting Case M odels}

A limiting case is when the reaction rate is fast enough and the reactants suф ciently abundant that any molecule of contaminant reaching the bubble surface will instantly disappear though a reaction. The reaction rate is thus limited by contaminant transport to the bubble surface and the total bubble surface area. This represents an upper limit on how rapidly a contaminant can disappear. For spherical, toroidal, and cylindrical bubble shapes, solution of eq. (9) for the contaminant concentration, $C$, with initial value $C_{0}$, exhibits an exponential decrease with time, t;(as shown below):

$$
\mathrm{C}=\mathrm{C}_{\mathrm{o}} \mathrm{e} \cdot \mathrm{t} ; \quad, \quad=\mathrm{B} \frac{\mathrm{S}_{\max }}{\mathrm{V}} \frac{\overline{\mathrm{D}}}{\mathrm{T}_{\mathrm{cz}}} ; \quad \mathrm{T}_{\mathrm{cz}}=\frac{\mathrm{V}_{\mathrm{cz}}}{\mathrm{Q}}:
$$

Here, $S_{\max }$ is the maximum bubble surface area, $V$ is the total fow loop liquid volume, $V_{c z}$ is the liquid volume in the cavitation zone, $\mathrm{Q}$ is the volumetric $\neq$ ow rate through the loop, and $\mathrm{D}$ is the dixusivity of the contaminant in the liquid. The time scale $T_{c z}$ is the liquid mean residence time in the cavitation zone $B$ is a constant that depends on the speci..c bubble geometry. 

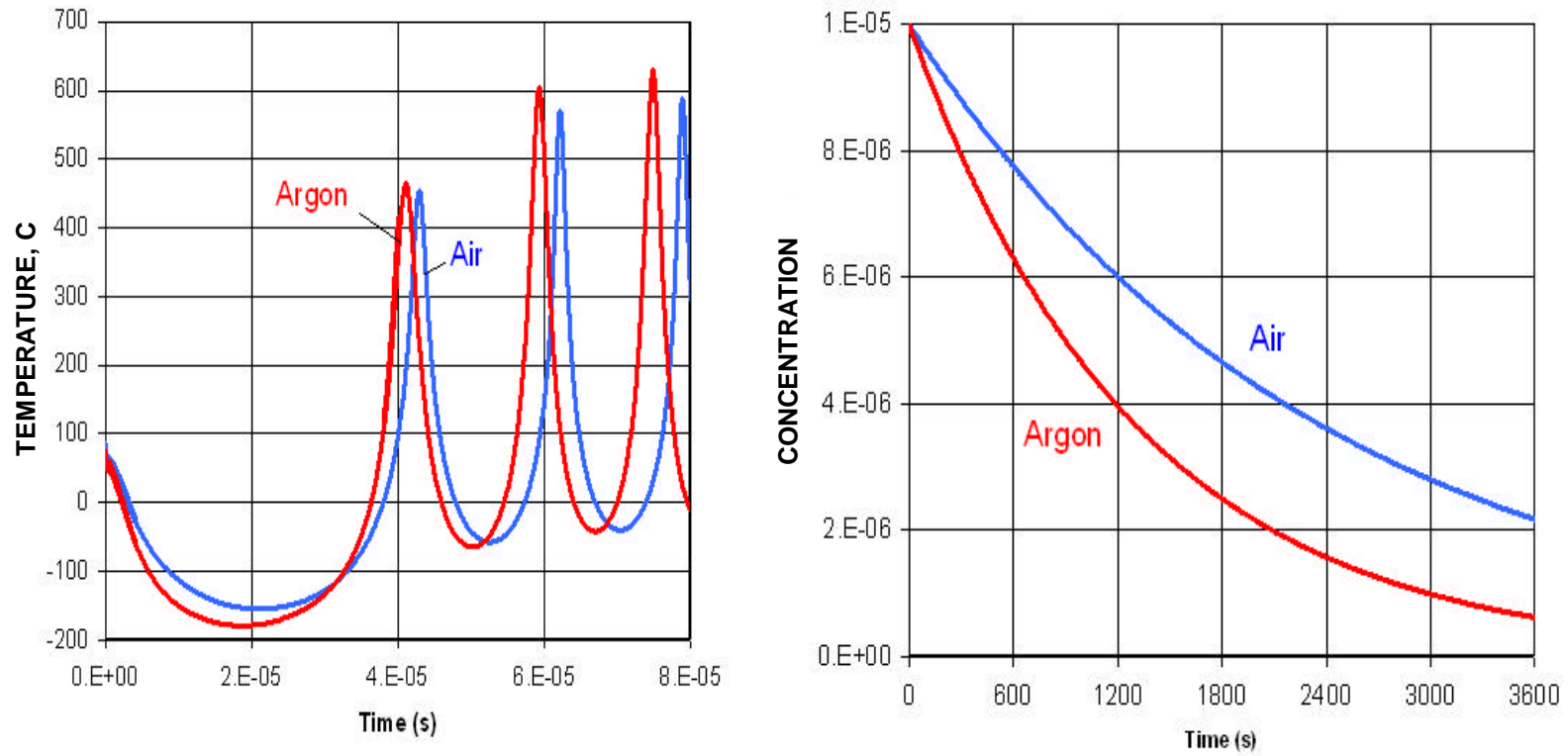

Figure 7: Calculated inłuence of type of noncondensible gas (air and Argon) within bubble on oxidation of PNP. Left: temperature within bubble Right: Concentration history.
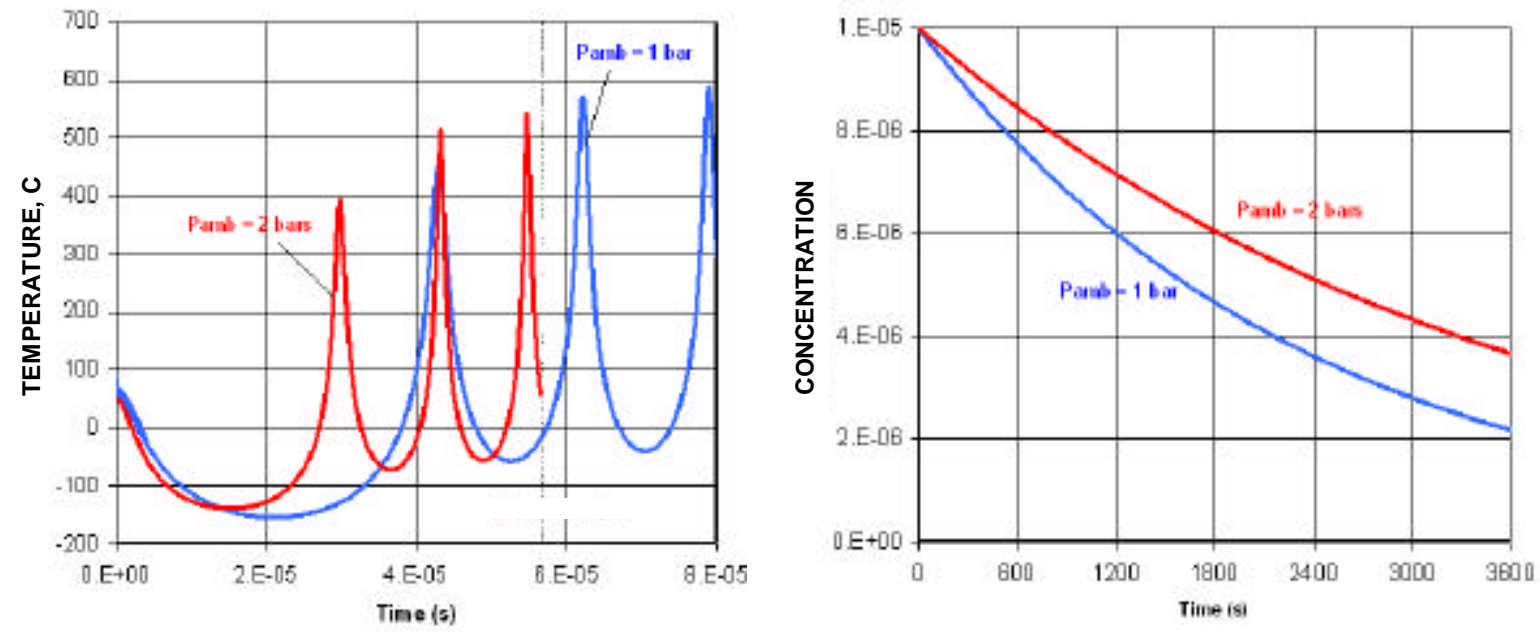

Figure 8: Computed bubble dynamics in the łow ..eld of a submerged jet with mass and heat transfer: infuence of ambient pressure on bubble temperature history and oxidation of contaminant. Cavitation Number $=0.25$, ambient temperature $=50{ }^{ \pm} C$, initial bubble size $=0.1 \mathrm{~mm}$.

Spherical Bubble

For a single spherical bubble, expression (6) for mass $\ddagger u x$ becomes:

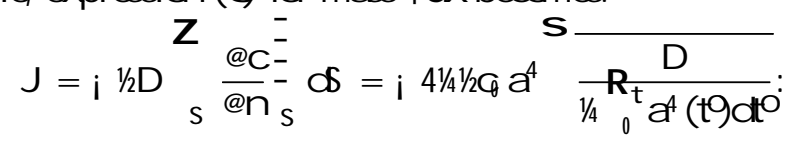


For a multiphase medium,

$$
\eta \mathrm{i}=i \quad 8 / 2 / \varepsilon_{0} \frac{\mathrm{D}}{\frac{D}{1 / 4} \operatorname{ra}^{4} \mathrm{i}:}
$$

We..nd that expression (9) simpli..es for h®i $1 / 40$ (small void fraction) to (10) with

$$
B=p_{1 / 4}^{2} ; \quad S_{\max }=4 / / m a_{\max }^{2}:
$$

We evaluated these expressions and compared with experimental data for PNP oxidation using the 216 1-mm ori..ce nozzle ( $\$ P=60$ psi, $P_{a m b}=1 \mathrm{~atm}, Q=54 \mathrm{gpm}, V=7.9$ liters, $r_{v}=0.0003, D=2: 4 f 10^{9}$ $\mathrm{m}^{2}=\mathrm{s} ; \mathrm{T}_{\text {loop }}=2.3 \mathrm{~s}$ ). The value of the decay constant was found to be: $0.09 \cdot, \cdot 0.024 \mathrm{~min}^{\mathrm{i}}{ }^{1}$ : This was calculated from the measured concentrations over the time period needed for a factor of ten reduction in concentration (which varied between 25 and $95 \mathrm{~min}$.) We set $n=216 n^{\alpha}$; where $n^{\alpha}$ is the average number of bubbles, of maximum radius $a_{\max }$, simultaneously present for each of the 216 nozzles. Using these values in expressions (10) and (13), the calculated values of are found to be within this experimental range for $\mathrm{n}^{\mathrm{x}}=2,3$, and 4 when $\mathrm{a}_{\max }=0.5 \mathrm{~mm}$ (the ori..ce radius) and for $\mathrm{n}^{\mathrm{x}}=1$ and 2 when $\mathrm{a}_{\max }=1 \mathrm{~mm}$ (twice the ori..ce radius).

Toroidal Bubble

If the cavities are considered to be toroids with major diameter, $D_{\text {ring, }}$, and minor (cross-sectional) diameter, $d_{\text {ring; }}$ the surface area $S$ is given by:

$$
\mathrm{S}=1 / 2 \mathrm{D}_{\text {ring }}^{2} \frac{\mathrm{d}_{\text {ring }}}{\mathrm{D}_{\text {ring }}}
$$

and $B$ remains the same as in (13). From observation, $D_{\text {ring }}$ is of the order of 1 to 2 times the ori..ce diameter, and thus 0.1 to $0.2 \mathrm{~cm}$ for the cases considered. Results of evaluation of relation (10) that yield predicted values of , within the measured range for values of $n^{\alpha}$ of 3 or less (consistent with observations) show that the ratio $d_{\text {ring }} \Rightarrow_{\text {ring }}$ should be in the range $0.2-0.3$. The presence of 1 to 3 rings is typical in the experiments as are these values of $D_{\text {ring; }}$;and $d_{\text {ring }}=D_{\text {ring }}$ :

The above analysis and evaluations suggest that, at least within the parameter range of the listed experiments, the jet oxidation process may be controlled by transport of contaminants to cavity surfaces and be relatively insensitive to radical generation rate and the rate of the kinetics of the oxidation reaction.

\section{Oxidation in a Swirl Flow with a Gaseous Core}

We derived a limiting case expression for dixusion to a central line vortex cavity to model contaminant transport to the cavity in the swirl chamber of the swirling cavitating jet. The reaction is considered to take place immediately as the contaminant reaches the cavity surface. We model the swirling jet fow as steady and axisymmetric about a central gaseous modeled as an in. .nite cylinder of constant radius a: We consider a uniform angular (tangential) fow velocity near the cylinder and an axial łow paralle to the cylinder and assume that these components of the velocity depend only on the radial distance $r$. The transport of a dissolved compound of concentration c to the cylindrical core can be described by the convection-dixusion equation. For the limiting case of in. .nite reaction rate and for a thin diausion boundary layer, we...nd:

$$
c=c_{1} \text { erf } \frac{\tilde{A} i a}{2} \frac{r}{\frac{U}{D z}} ; \quad J=i^{1 / \mathbb{k}_{1}}{ }_{1 / 2}^{p} \frac{\overline{I D U}}{\ln }
$$

where $R$ is the radius of the boundary of the swirl chamber, $I$ is the cavity length, $U$ the average axial velocity computed from the fow rate and the cross sectional area of the swirl chamber, and $z$ is the axial distance from the upstream end of the cavity. We ..nd that (10) again applies with:

$$
\mathrm{S}_{\max }=21 / \mathrm{aL}_{\mathrm{sc}} ; \quad \mathrm{B}=1 ; \mathrm{V}_{\mathrm{cz}}=\mathrm{V}_{\mathrm{sc}} ;
$$

where $V_{s c}$ is the swirl chamber volume, and $L_{s c}$ is the swirl chamber length - or total cavity length within the chamber. 
The values of , wereevaluated for various swirling jet cases for 1 hour of operation $\left(D=2: 4 f 10^{i} \mathrm{~cm}^{2} \Rightarrow\right.$, $V=8$ liters, a $1 / 4$ 0:25 cm) and are plotted versus the fow rate in Figure 9 . The data have been ..t with a power relation that provides a best-..t value of 0.5 for the power of $\mathrm{Q}$. The predicted values are low by a factor of 5 to 10 relative to the data. This may be due to contaminant transport mechanisms, such as bulk transport due to recirculation and turbulent transport, not considered in the model. The value of $D$ used in evaluating expression (10) was the laminar fow molecular dixusivity equal to $2.4 \mathrm{f} 10^{i} \mathrm{~cm}^{2} / \mathrm{s}$ which we de. ne as $D_{0}$. If $D$ is replaced with a turbulent dixusivity 33 times the molecular value, the predictions match the "best ..t" power relation of the data. This is not an unreasonable value for turbulent diausivity.

Lambda vs. Flow Rate, $\mathbf{Q}$

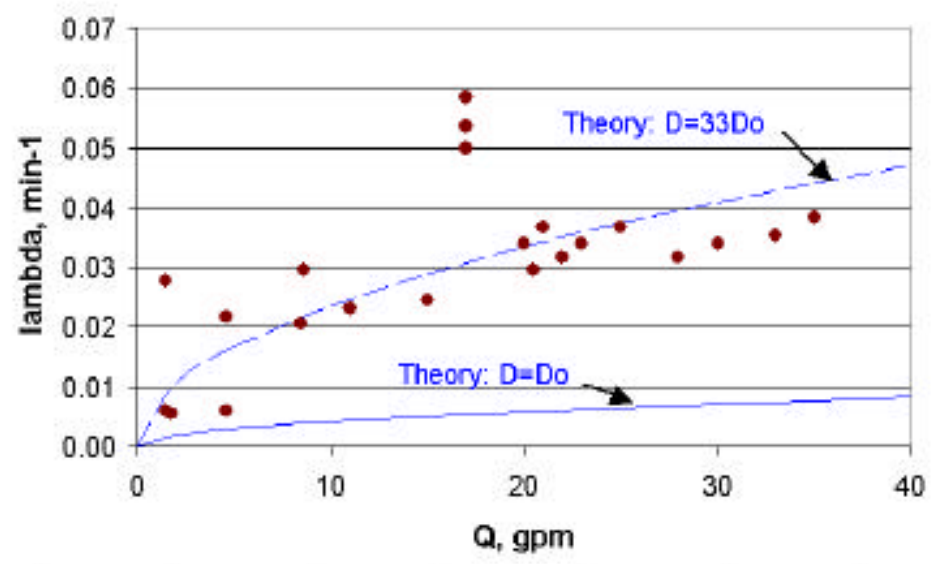

Figure 9: Comparison of measured and computed concentration decay constants, lambda, for swirling cavitating jet oxidation of Methyl Orange for $60 \mathrm{~min}$. of operation. $D_{0}=2: 4 \pm 10^{i} \mathrm{~cm}^{2}=$, the laminar fow dixusivity.

\section{Summary \& Conclusions}

Experiments and modeling on the use of jet induced cavitation to oxidize dilute aqueous solutions of organic compounds were performed Experiments with p-Nitrophenol and Methyl Orange demonstrated the ability of these jets to rapidly reduce the concentrations of both compounds below $1 \mathrm{ppm}$ (the lowest value we could accurately measure with our instrumentation) and to do so with more that a two order of magnitude increase in energy ec ciency compared to an ultrasonic device

Additional ...ndings include:

2 Highest ed ciencies were obtained with the lowest fow rates and pressures.

2 The ec ciency increases with increase in the total cavity surface area due to a large number of small ori..ces.

2 Analysis indicated the reactions were limited by contaminant transport to bubble surfaces.

2 Operation at elevated ambient pressures was not found to result in more rapid oxidation. Thus from an eq ciency standpoint, it is desirableto operatenear atmospheric ambient pressure. This is unanticipated in that it is well known that higher ambient pressures produce moreintensebubblecollapse. A potential explanation is that the jet cavitation collapse at atmospheric pressure produces suఢ cient radicals such that an increase in intensity does not change the oxidation rates.

\section{A cknowledgments}

The authors wish to thank Dr. Nail Gumerov of Dynaf I ow for discussions and modeling exorts and Dr. Lawrence Principe of J ohns Hopkins University for a number of helpful discussions and consultations. This work was sponsored in part by NSF under SBIR award No. DMI-9661572. 


\section{R eferences}

Brown, B. and Goodman, J . E., 1965, High Intensity Ultrasonics, Van Nostrand Inc., Princeton, NJ .

Chahine, G.L. and Genoux, Ph., 1983, "Collapse of a Cavitating Vortex Ring," J ournal of Fluids Enginering Vol. 105, pp. 400-405.

Chahine G.L., and J ohnson V. E.. J r., 1985, “Mechanics and A pplications of Self-Resonating Cavitating J ets," International Symposium on J ets and Cavities, ASME, WAM, Miami, FL.

Chahine, G. L. \& Kalumuck, K. M., 2001a, "Fluid J et Cavitation Method and System for E\$ cient Decontamination of Liquids," U.S. Patent No. 6,200,486.

Chahine, G. L. \& Kalumuck, K. M., 2001b "Swirling Fluid J et Cavitation Method and System for E $\varnothing$ cient Decontamination of Liquids," U.S. Patent No. 6,221,260.

Chahine, G.L., Kalumuck, K. M., \& Frederick, G. S., 1995, “Cavitating Water J ets for Deep Hole Drilling in Hard Rock," Proc., 8th American Water J et Conference, Houston, TX, Vol. 2, pp. 765-778.

Cheung, H. M., Bhatnagar, A. and J ansen, G., 1991, "Sonochemical Destruction of Chlorinated Hydrocarbons in Dilute Aqueous Solution," Env. Sci. \& Techn., Vol. 25, pp. 1510.

Genoux, Ph. and Chahine, G.L., 1984, "Simulation of the Pressure Field Due to a Submerged Oscillating J et Impacting on a Solid Wall," J ournal of Fluids Engineering, Vol. 106, pp. 491- 496.

Gong, C. and Hart, D. P., 1998, "Ultrasound Induced Cavitation and Sonochemical Y ields," J ournal of the Acoustical Society of America, vol. 104, No. 4, pp. 2675-2682.

Gumerov, N. A., 2000, "Dynamics of Vapor Bubbles with Non-Equilibrium Phase transitions in Isotropic Acoustic Fields," Physics of Fluids, vol 12, No. 1.

Hua, I., Hochemer, R., and Hoxman, M., 1995a, "Sonochenmical Degradation of p-Nitrophenol in a Paralle Plate Near Field Acoustic Processor," Env. Sci. \& Techn., Vol. 29, pp. 2790-2796, .

Hua, I., Hochemer, R., and Hoxman, M., 1995b, "Sonolytic Hydrolysis of p-Nitrophenyl Acetate: The Role of Supercritical Water," J ournal of Physical Chemistry, Vol. 99, pp. 2335-2342.

Hua, I. and Hoxman, M., 1996, "Kinetics and Mechanism of the Sonolytic Degradation of $\mathrm{CCl}_{4}$ : Intermediates and Byproducts," Env. Sci. \& Techn., Vol. 30, pp. 864-871.

Kalumuck, K. M. \& Chahine, G. L., 2000, "The Use of Cavitating J ets to Oxidize Organic Compounds in Water," J ournal of Fluids Engineering, Vol. 122, pp.465-470. (Also in Proceedings, ASME Fluids Engineering Summer Meeting, Washington D.C., ASME FEDSM98-4813, J une 1998.)

Kalumuck, K. M., Chahine, G. L., Gumerov, N. A., Aley, P. D., \& Frederick, G. S., 2000, “Oxidation of Organic Compounds in Water with Cavitating J ets - Phase II" Dynaf I ow, Inc. Tech. Rpt. 98010.

Kotronarou, A., Mills, G., and Hoxman, M., 1991, "Ultrasonic Irradiation of p-Nitrophenol in Aqueous Solution," J ournal of Physical Chemistry, Vol. 95, pp. 3630-3638.

Neppiras, E. A., 1980, “Acoustic Cavitation, " Phys. Rep., 61, 159-251.

Perry, R. \& Green, D., eds., 1984, Perry's Chemical Engineers' Handbook, 6th ed., McGraw-Hill, NY.

Plesset, M. S. and Zwick, S. A., 1952, "A Nonsteady Heat Diqusion Problem with Spherical Symmetry," J. Applied Physics, vol. 23, No. 1.

Skov, E., Pisani, J . and Beale, S., 1997, "Cavitation Induced Hydroxyl Radical Formation," American Institute of Chemical Engineering National Meeting, Houston,TX.

Suslick, K. S., ed., 1988, Ultrasound, Its Chemical, Physical, and Biological Exects, VCH, New York.

Suslick, K. S., Cline, J r., R. E., and Hammerton, D.. A., 1986, "The Sonochemical Hot Spot," J ournal of the American Chemical Society, Vol. 108, p. 5641.

Suslick, K. S., 1989, "Sonochemistry," Science, Vol. 247, pp. 1439-1445.

Young, F. R., 1989, Cavitation, McGraw-Hill, London. 
